\title{
Optical Heterodyne Saturation Spectroscopy in Ammonia Filled Hollow-Core Photonic Bandgap Fibers
}

\author{
Ana M. Cubillas ${ }^{1,2}$, Jan Hald ${ }^{1}$, and Jan C. Petersen ${ }^{1}$ \\ ${ }^{1}$ Danish Fundamental Metrology Ltd., Matematiktorvet 307, DK-2800 Kgs. Lyngby, Denmark \\ ${ }^{2}$ Photonics Engineering Group, University of Cantabria, Avda. Castros s/n, 39005 Santander, Spain. \\ jcp@dfm.dtu.dk
}

\begin{abstract}
We have applied the frequency-modulation (FM) technique in the $1.5 \mu$ mavelength region to observe saturated absorption in ammonia in hollow-core photonic bandgap fibers (HCPBFs). Previously blended lines have been resolved. @2008 Optical Society of America OCIS codes: (060.5295) Photonic crystal fibers, (300.6460) Saturation Spectroscopy.
\end{abstract}

HC-PBFs allow the confinement of high intensity light over long absorption paths. In this way, saturated absorption spectroscopy can be performed in these fibers [1-3]. When combined with FM spectroscopy, high sensitivities in the detection of weak molecular transitions can be achieved [4,5]. In this paper, we report the observation of FM saturation spectroscopy on the near-infrared $v_{1}+v_{3}$ band of ammonia $\left({ }^{14} \mathrm{NH}_{3}\right)$ in a HC-PBF. To the best of our knowledge, this is the first observation of sub-Doppler spectroscopy on ammonia in the near-infrared.

The experimental setup used for FM saturation spectroscopy is shown in Fig. 1. The pump (red beam) is amplitude-modulated by an acousto-optical modulator at a frequency of $91 \mathrm{KHz}$ and shifted $40 \mathrm{MHz}$ in frequency. The probe (green beam) is phase-modulated by an electro-optic modulator at a frequency of $54 \mathrm{MHz}$. The 3.5 meter HC-PBF used in the experiment was produced by Crystal Fibre A/S (type HC19-1550-01 with 20- $\mu$ m diameter core). The signal is recovered by double phase-sensitive detection using a radio frequency mixer and a lock-in amplifier.

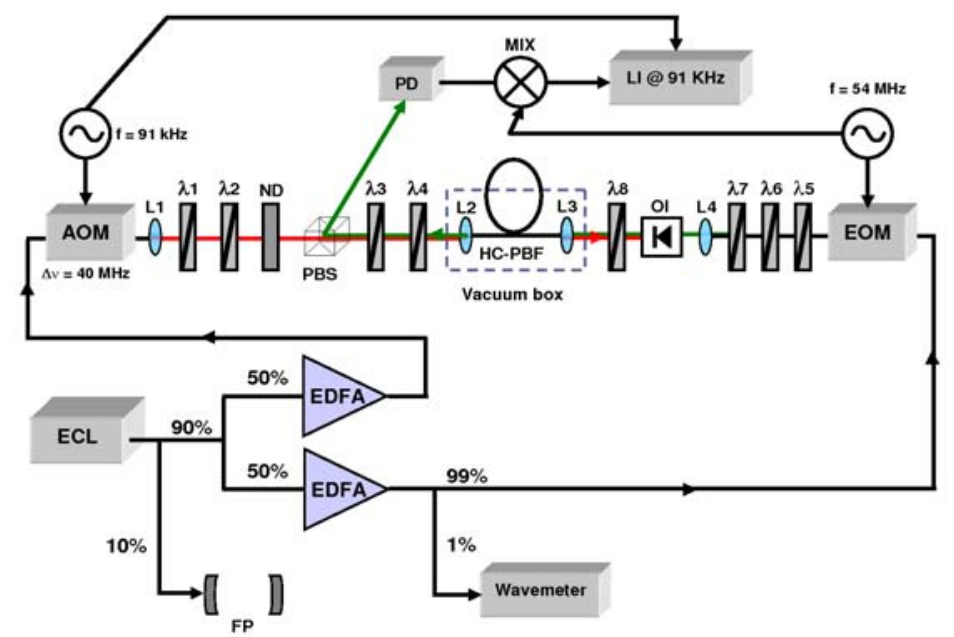

Fig. 1. Experimental setup for FM saturation spectroscopy with External Cavity Laser (ECL), Erbium Doped Fiber Amplifiers (EDFAs), FabryPerot resonator (FP), collimating lenses L1-L4, wave plates $\lambda_{1}-\lambda_{8}$, acousto-optical modulator (AOM), electro-optic modulator (EOM), Neutral Density Filter (ND), Optical Isolator (OI), Polarizing Beam Splitter (PBS), Photo Detector (PD), Radio Frequency Mixer (MIX) and Lock-In amplifier (LI).

The sub-Doppler signal recovered by the system is described by the following equation [5]:

$$
I_{F M}(v)=\alpha\left[\left(L^{-}-L^{+}\right) \sin \phi+\left(D^{+}-2 D^{0}+D^{-}\right) \cos \phi\right]
$$

Where, $\alpha$ is a scaling factor, $\phi$ is the phase shift between the local oscillator and the mixer and $L^{j}$ and $D^{j}$ are two profile functions defined as follows:

$$
L^{j}=\frac{\Gamma^{2}}{\Gamma^{2}+\left(v^{j}-v_{0}\right)^{2}} \quad \text { and } \quad D^{j}=\frac{\Gamma\left(v^{j}-v_{0}\right)}{\Gamma^{2}+\left(v^{j}-v_{0}\right)^{2}}
$$


In which, $v$ is the laser detuning, $v_{0}$ is the center of the resonance, superscript $j$ can have three values $j=+, 0,-$, which denote the three FM components $v^{ \pm}=v \pm f m / 2$ and $v^{0}=v$, respectively, $f m$ is the modulation frequency and $\Gamma$ is the linewidth (HWHM) of the resonance.

Two examples of measured FM saturation signals are shown in Fig. 2. They have been fitted using the expression in Eq. 1. The fits are inserted in the upper parts of the figures while the residuals of the fits are shown in the lower parts. The line shown to the left in Fig. 2 is the ${ }^{\mathrm{P}} \mathrm{P}(4,3)^{\mathrm{a}}$ transition of the $v_{1}+v_{3}$ band at $1526.9976 \mathrm{~nm}$. The lines at $1531.6815 \mathrm{~nm}$ and $1531.6824 \mathrm{~nm}$ in the right part of Fig. 2 are two previously unresolved transitions in the $v_{1}+v_{3}$ band (transitions ${ }^{\mathrm{P}} \mathrm{P}(6,6)^{\mathrm{a}}$ and ${ }^{\mathrm{P}} \mathrm{P}(5,3)^{\mathrm{a}}$, respectively) [6]. These two transitions can only be resolved using sub-Doppler spectroscopy. As can be seen, an excellent agreement between the theory and the experiment is demonstrated. The line widths of the three lines, which are mainly due to self-pressure broadening, are found to be 29, 26 and $30 \mathrm{MHz}$ (HWHM) in good agreement with previously reported collision broadening rates [7].

Applications for this work include high-resolution spectroscopy and optical frequency standards based on lasers stabilized to molecular absorption lines.
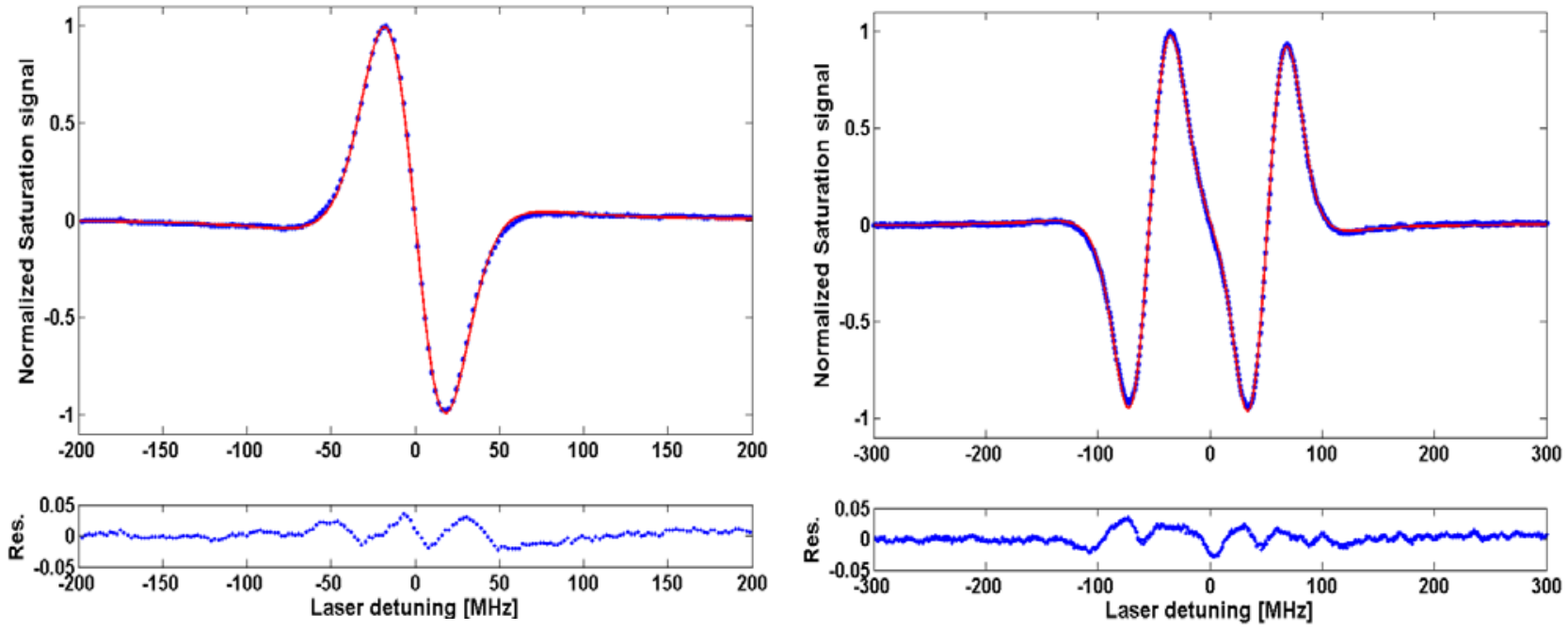

Fig. 2. FM saturation signals of ${ }^{14} \mathrm{NH}_{3}$ in a 3.5-m HC-PBF at a pressure of 2 mbar. Blue dots represent the measured signal and solid red line the obtained fit. The residual is shown in the bottom panel.

\section{References}

[1] J. Henningsen, J. Hald and J. C. Petersen, " Saturated absorption in acetylene and hydrogen cyanide in hollow core photonic bandgap fibers," Opt. Express 13, 10475-10482 (2005).

[2] R. Thapa, K. Knabe, M. Faheem, A. Naweed, O. L. Weaver and K.L. Corwin, "Saturated absorption spectroscopy of acetylene gas inside large-core photonic bandgap fiber,” Opt. Lett. 31, 2489-2491 (2006).

[3] J. Hald, J. C. Petersen and J. Henningsen, "Saturated Optical Absorption by Slow Molecules in Hollow-Core Photonic Band-Gap Fibers”, Phys. Rev. Lett. 98, 213902 (2007).

[4] G. C. Bjorklund, "Frequency-modulation spectroscopy - new method for measuring weak absorptions and dispersions," Opt. Lett. 5, 15-17 (1980).

[5] J. L. Hall, L. Hollberg, T. Baer and H.G. Robinson, “Optical heterodyne saturation spectroscopy”, Appl. Phys. Lett. 39, 680-682 1981.

[6] L. Lundsberg-Nielsen, F. Hegelund and F.M. Nicolaisen, ”Analysis of the high-resolution spectrum of ammonia $\left({ }^{14} \mathrm{NH}_{3}\right)$ in the nearinfrared region, 6400-6900 $\mathrm{cm}^{-1}$ )," J. Mol. Spectros. 162, 230-245 (1993).

[7] A. Lucchesini and S. Gozzini, "Collisional broadening and shifting of ammonia absorption lines at 790 nm,” Eur. Phys. J. D 22, 209215 (2003). 